\title{
Green processing of porous chitin structures for biomedical applications combining ionic liquids and supercritical fluid technology
}

\author{
Simone S. Silva ${ }^{\mathrm{a}, \mathrm{b}, *}$, Ana Rita C. Duarte ${ }^{\mathrm{a}, \mathrm{b}}$, Ana Paula Carvalho ${ }^{\mathrm{c}}$, João F. Mano ${ }^{\mathrm{a}, \mathrm{b}}$, Rui L. Reis ${ }^{\mathrm{a}, \mathrm{b}}$ \\ a 3 B́s Research Group - Biomaterials, Biodegradables and Biomimetics, Department of Polymer Engineering, University of Minho, \\ Headquarters of the European Institute of Excellence on Tissue Engineering and Regenerative Medicine, AvePark, Zona Industrial da Gandra, \\ Caldas das Taipas, 4806-909 Guimarães, Portugal \\ ${ }^{\mathrm{b}}$ IBB - Institute for Biotechnology and Bioengineering, PT Associated Laboratory, Portugal \\ ' University of Lisbon, Department of Chemistry and Biochemistry, Faculty of Science, Campo Grande, Ed. C8, 1749-016, Lisboa, Portugal
}

\section{A R T I C L E I N F O}

\section{Article history:}

Received 10 May 2010

Received in revised form 17 September

2010

Accepted 30 September 2010

Available online 8 October 2010

\section{Keywords:}

Biomaterials

Chitin

Ionic liquids

Supercritical drying

Biomedical applications

\begin{abstract}
A B S T R A C T
The application of green chemistry principles in the processing of materials for advanced technologies is a steadily increasing field of research. In this work porous chitin-based materials were developed by combining the processing of chitin using ionic liquids (ILs) as a green solvent together with the use of supercritical fluid technology (SCF) as clean technology. Chitin was dissolved in 1-butyl-3-imidazolium acetate, followed by regeneration of the polymer in ethanol in specific moulds. The IL was removed using Soxhlet extraction and successive steps of extraction with SCF using carbon dioxide/ethanol ratios of 50/ 50 and 70/30. The developed porous chitin-based structures (ChIL) can be classified as mesoporous materials, with very low density and high porosity. The cytotoxicity of ChIL extracts was investigated using L929 fibroblast-like cells, and the results demonstrated that the produced materials have extremely low cytotoxicity levels. Therefore, the findings suggest that the porous chitin structures may be potential candidates for a number of biomedical applications, including tissue engineering.
\end{abstract}

( 2010 Acta Materialia Inc. Published by Elsevier Ltd. All rights reserved.

\section{Introduction}

In recent years, natural renewable resources have been successfully used to produce biomaterials that are biodegradable under certain temperature and humidity conditions. Unfortunately, most biodegradable materials are synthesized by chemical processes using volatile organic solvents, and their emissions and disposal are a large source of pollution worldwide. Alternative solvents such as ionic liquids (ILs), also known as "green solvents", have emerged as a class of solvents that can be used as components of polymeric matrices, templates for porous polymers and solvents for a wide variety of organic and inorganic compounds [1,2]. By definition, ILs are organic salts that are liquids at room temperature and exhibit unique physicochemical properties, namely no measurable vapor pressure, excellent chemical and thermal stabilities, high ionic conductivity and easy recyclability [3]. Based on these properties, the use of ILs as solvents or nonvolatile reaction media instead of conventional

\footnotetext{
* Corresponding author at: 3B́s Research Group - Biomaterials, Biodegradables and Biomimetics, Department of Polymer Engineering, University of Minho, Headquarters of the European Institute of Excellence on Tissue Engineering and Regenerative Medicine, AvePark, Zona Industrial da Gandra, Caldas das Taipas, 4806-909 Guimarães, Portugal. Tel.: +351 253510900; fax: +351 253510909.

E-mail address: simonesilva@dep.uminho.pt (S.S. Silva).
}

organic solvents can minimize a number of environmental and safety problems [4]. Recently, ILs have also provided a new processing platform for the dissolution of some natural macromolecules (e.g. cellulose, chitin, starch and lignin) that are otherwise difficult to dissolve due to their inter- and intramolecular hydrogen bonds [1]. In the IL platform, macromolecules can be dissolved, regenerated, derived and functionalized, increasing their potential for exploitation. The pioneering work on ILs as solvents for polysaccharides was reported by Swatloski et al. [5] in 2002. They found that ILs can be used as non-derivatizing solvents for native cellulose. The success of dissolving cellulose in ILs [6,7] has initiated a series of studies on the dissolution of other biopolymers and/or the development of new ILs.

Polysaccharides such as chitin and chitosan present a wide diversity of structures and properties that allow several possible chemical and mechanical modifications in order to create novel materials with specific functionalities and properties [8-11]. Despite its widespread availability, the use of chitin has been limited due its intractability and insolubility in water and common organic solvents [8-11]. Nevertheless, the dissolution of chitin in ILs allows its processing into high added-value materials, e.g. porous structures. This has significantly broadened the number of tailored derivatives from this polysaccharide, and consequently enhances its potential use in a number of applications, including in the biomedical field. 
This work intends to apply the green chemistry principles [12] to the development of porous chitin-based materials through the combination of green solvents, such as ILs, used in the dissolution of chitin together with the use of a clean and environmentally friendly technology, namely supercritical fluid technology (SCF). The unique solvent tunability of supercritical fluids, from gas-like to liquid-like properties, offers the possibility of precise control over the processing conditions, which can be adapted in many ways for the extraction of ILs. With this in mind, chitin was dissolved in 1-butyl-3-methylimidazolium acetate ([bmim][Ac]), followed by regeneration of the polymer in ethanol in specific moulds. The [bmim][Ac] was removed using Soxhlet extraction and successive steps of extraction with carbon dioxide/ethanol $\left(\mathrm{CO}_{2} / \mathrm{EtOH}\right)$ ratios under near-critical conditions. Later on, the samples were dried with $\mathrm{CO}_{2}$ under supercritical fluid conditions, and porous chitin structures were produced. The characterization of the porous chitin structures was performed using different techniques. To investigate the possible cytotoxic effect of the developed materials, both chitin extracts and [bmim][Ac] solutions were placed in contact with fibroblast-like cells (L929) and a cellular viability assay (MTS) was performed.

\section{Materials and methods}

\subsection{Materials}

Chitin from crab shells (practical grade; Sigma Aldrich, CAS 1398-61-4) was ground through a Wiley Mill (model 4, Thomas) and stored in plastic bottles. Ground chitin $(106 \mu \mathrm{m})$ was used throughout all experiments to obtain reproducible results. Bulk density of chitin was determined following procedures described by Anderson et al. [13]. The IL [bmim][Ac] was from Sigma Aldrich and used as received. Carbon dioxide (99.998 mol \%) was supplied by Air Liquide. All other chemicals were reagent grade and used as received.

\subsection{Chitin solution preparation and shaping}

Chitin was dissolved in [bmim] $[\mathrm{Ac}]$ at $90-95^{\circ} \mathrm{C}$ for $5 \mathrm{~h}$ under stirring. Chitin-IL (ChIL) solutions were prepared at concentrations of $1-3 \%(w / v)$. After solubilization, chitin/IL solutions were transferred to specific moulds ( $3 \mathrm{ml}$ for each mould) and the system was left to age at $4{ }^{\circ} \mathrm{C}$ for $1 \mathrm{~h}$, then the moulds were immersed in ethanol for $24 \mathrm{~h}$ at room temperature to obtain chitin gels. The chitin gel formation was also tested by dissolving chitin in dimethylacetamide/lithium chloride (DMAc/LiCl) in the same concentrations and under the same conditions as used previously with the ILs.

\subsection{IL extraction}

The [bmim] $[\mathrm{Ac}]$ was removed from the gels through Soxhlet extraction with ethanol for 2 days and successive steps of supercritical drying $\left(\mathrm{CO}_{2}\right)$. Typically, a Soxhlet extraction is carried out when the compound to be extracted has a limited solubility in a solvent and the solute is insoluble in that solvent. The extraction cycle may be repeated many times, over hours or days. For IL removal, 2 days were necessary. During each cycle, a portion of the IL was dissolved in ethanol and after several cycles the polymeric material was purified. The advantage of this system was the fact that just one batch of solvent (ethanol) was used, which was recycled during the process to avoid using a large quantity. The SCF process removes the [bmim] $[\mathrm{Ac}]$ residues (step 1) and also promotes their drying (step 2). During step $1(3 \mathrm{~h}$ ), aliquots of ethanol were collected for analysis of conductivity. The conductivities of the aliquots of ethanol were measured using a conductivimeter (INOLAB, Multi level 3) with a Sonda WTW TetraCon 325. Extraction of the ionic liquid was performed in a high-pressure facility, schematically represented in Fig. 1. Briefly, the samples are loaded into the high-pressure vessel, which is heated by means of an electric thin band heater (OGDEN) connected to a temperature controller. The temperature was maintained within $\pm 1^{\circ} \mathrm{C}$. The desired mixture of carbon dioxide + ethanol was pumped into the vessel using a high-pressure piston pump (P-200A Thar Technologies) for the carbon dioxide and a liquid pump (P-50 Thar Technologies) for the ethanol. The two solvents were mixed in a static mixer (SM) and heated to the operating temperature before entering the reactor $(\mathrm{HE})$. The pressure inside the vessel was measured with a pressure transducer $(P)$. The carbon dioxide + ethanol flow was regulated by a flow meter (FM; Siemens, SITRANS FC MASS FLO MASS2100) and the outflow was regulated using a metering valve. To optimize the ionic liquid extraction, two carbon dioxide/ethanol $\left(\mathrm{CO}_{2} / \mathrm{EtOH}\right)$ ratios were used, namely $70 / 30$ and $50 / 50 \mathrm{CO}_{2} / \mathrm{EtOH}$, at near-critical conditions.

\subsection{Sample drying}

Supercritical fluid drying was performed with the same apparatus and following the same procedure. However, a stream of pure carbon dioxide was flushed into the system at a constant flow rate $\left(5 \mathrm{~g} \mathrm{~min}^{-1}\right)$. The critical drying step typically took $1 \mathrm{~h}$. Both processes (IL extraction and drying) were performed with a pressure of $100 \mathrm{bar}$ and a temperature of $70^{\circ} \mathrm{C}$. The porous chitin structures produced were identified as ChIL70 and ChIL50, corresponding to $70 / 30$ and $50 / 50 \mathrm{CO}_{2} /$ EtOH ratios used in the SCF. The porosity of the obtained materials was estimated by the following equation [14]:

$P=\left(1-d_{\mathrm{p}} / d_{\mathrm{b}}\right) \times 100$

Where $d_{\mathrm{p}}$ is the density of the porous material and $d_{\mathrm{b}}$ is the density of the bulk material. The density of the porous samples was calculated by measuring their mass and their dimensions.

\subsection{Characterization}

\subsubsection{Fourier transform infrared (FTIR) spectroscopy}

The infrared spectra of the chitin structures were obtained with a Shimadzu-IR Prestige 21 spectrometer in the spectral region of $4000-650 \mathrm{~cm}^{-1}$ with a resolution of $2 \mathrm{~cm}^{-1}$ for 32 scans. The samples were powered, mixed with $\mathrm{KBr}$ and processed into pellets.

\subsubsection{Scanning electron microscopy (SEM)}

The morphology of the samples coated with gold was obtained at $10 \mathrm{kV}$ on a NanoSEM-FEI Nova 200 equipped with an energydispersive spectrometer for silicon microanalysis.

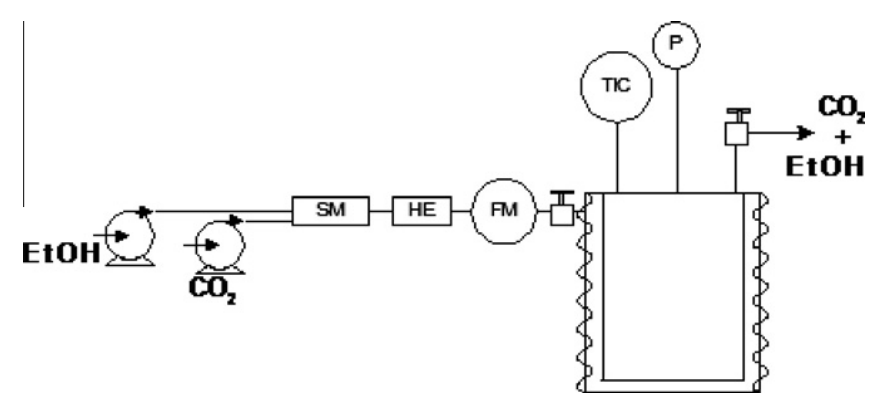

Fig. 1. Schematic representation of the high-pressure apparatus (SM, static mixer; $\mathrm{HE}$, heat exchanger; FM, flow meter; TIC, temperature controller; P, pressure transducer). 


\subsubsection{Determination of superficial area}

$\mathrm{N}_{2}$ adsorption isotherms of the chitin samples at $-196{ }^{\circ} \mathrm{C}$ were measured in an automatic apparatus Micromeritics ASAP 2010. Before the isotherms acquisition, the samples $(\approx 250 \mathrm{mg})$ were degassed for $4 \mathrm{~h}$ at $150^{\circ} \mathrm{C}$ under a vacuum of better than $10^{-2} \mathrm{~Pa}$. The values of the specific surface areas, $S_{\mathrm{BET}}$, were assessed applying the BET equation (in the range $0.05<p / p^{0}<0.35$ ) [15]. The mesopore size distribution was made according the Broekhoff-de Boer method, in a version simplified with the Frenkel-Halsey-Hill equation [16].

\subsubsection{X-ray diffraction (XRD)}

XRD patterns of the chitin samples were collected on a Bruker AXS D8 Discover diffractometer $(40 \mathrm{kV}, 40 \mathrm{~mA})$ in a $\theta / 2 \theta$ scan regime, using Ni-filtered $\mathrm{Cu} K_{\alpha 1,2}$ radiation, step $0.04^{\circ}$, time per step $1 \mathrm{~s}$ and $2 \theta$ range $4-40^{\circ}$. Crystalline values were calculated using WinPLOTR software.

\subsubsection{Mechanical properties}

Compressive mechanical properties of the developed chitin structures were measured using an INSTRON 5540 load frame (Instron Int. Ltd., High Wycombe, UK). The compressive modulus is defined as the initial linear modulus on the stress-strain curves. The data presented were the result of the average of at least five measurements.

\subsubsection{Cytotoxicity studies}

Prior to cell culture studies, all porous chitin structures (ChIL) were sterilized under an ethylene oxide atmosphere. In order to assess the eventual cytotoxicity of the developed ChIL structures, extracts of the materials and [bmim] [Ac] solutions were prepared and placed in contact with the mouse fibroblast-like cell line L929, and tested using an MTS (3-(4,5-dimethylthiazol-2-yl)5-(3-carboxymethoxyphenyl)-2-(4-sulfophenyl)-2H-tetrazolium) assay in accordance with the protocols described in ISO/EN 10,993 [17]. Cells were cultured in basic medium: Dulbecco's modified Eagle's medium (DMEM; Sigma-Aldrich, USA) with phenol red supplemented with $10 \%$ foetal bovine serum (FBS, Gibco, UK) and $1 \%$ antibiotic/antimycotic (A/B, Gibco, UK) solution. L929 cells were incubated at $37{ }^{\circ} \mathrm{C}$ in an atmosphere containing $5 \%$ of $\mathrm{CO}_{2}$ until $90 \%$ confluence was achieved. Then a cell suspension was prepared with a concentration of $4 \times 10^{3}$ cells $\mathrm{ml}^{-1}$, which was seeded onto 96-well plates. 2929 cells were incubated for $24 \mathrm{~h}$. ChIL extracts were prepared as described previously [18]. The IL [bmim][Ac], previously diluted at predetermined concentrations $\left(1.68 \times 10^{-2}\right.$, $8.39 \times 10^{-3}, 2.10 \times 10^{-3}, 1.69 \times 10^{-3}$ nd $\left.4.23 \times 10^{-3} \mathrm{~g} \mathrm{ml}^{-1}\right)$ in culture medium, was added to the wells. The relative viability (\%) of the L929 cells was determined for each ChIL extract and compared to latex extracts, used as a positive control of cell death. After $24 \mathrm{~h}$, both ChIL extracts and IL solutions were removed, and the cells were washed in a phosphate-buffered saline solution (Sigma-Aldrich, USA). An MTS test was then performed to assess the viability of the L929 cells in contact with extracts. For this assay, an MTS solution was prepared using a 1:5 ratio of MTS reagent and culture medium containing DMEM without phenol red, 10\% FBS and $1 \% \mathrm{~A} / \mathrm{B}$ solution, followed by an incubation period $(3 \mathrm{~h})$ at $37{ }^{\circ} \mathrm{C}$. Finally, the optical density was read at $490 \mathrm{~nm}$ on a multiwell microplate reader (Synergy HT, Bio-Tek Instruments). All cytotoxicity screening tests were performed using six replicates.

\section{Results and discussion}

The applications of ILs in polymer science, e.g. as solvents for macromolecules, have many advantages since they allow dissolution, without derivatization, at high concentrations ( $>10 \mathrm{wt} . \%)$. This dissolution occurs due to the ILs' ability to disrupt the hydrogen bonds of the polymers. The polymer can be easily regenerated from IL solutions by the addition of water, ethanol or acetone $[1,2]$. In our experiments, an imidazolium-based IL, [bmim][Ac], was chosen as the solvent for chitin. The class of imizadolium cation-based ILs has proven to be highly attractive and versatile [3]. However, information on the physicochemical properties of [bmim][Ac] is scarce. In our experiments, chitin/IL solutions were prepared from the dissolution of chitin powder in [bmim][Ac] at concentrations from 1 to $3 \% \mathrm{w} / \mathrm{v}$. It was observed that the viscosity of the chitin/ IL solutions as well as dissolution time increased with increasing polymer concentration, and that $3 \% \mathrm{w} / \mathrm{v}$ is the highest chitin concentration that can be used for processing chitin-based materials. The chitin/IL solutions were transferred to specific moulds, where low-temperature $\left(4^{\circ} \mathrm{C}\right)$ aging and the immersion of the moulds in ethanol promoted the gelification of the system, and consequently the formation of chitin gels, with very good consistency. Compared to gels obtained from chitin/IL, the dissolution test with $\mathrm{DMAc} / \mathrm{LiCl}$ required a longer dissolution time, and the resulting solutions did not allowed the formation of similar chitin/IL gels. The obtained chitin/IL solution can be moulded into a range of shapes and sizes for various applications (e.g. membranes, cylinders packed monolithic columns). Generally, porous natural materials with nano- and macrosize pores have been produced by means of freeze-drying [19], supercritical $\mathrm{CO}_{2}$ [20-23] and melt-based processing methodologies [24], among others [25,26]. However, the success of these methods depends on the characteristics of the polymeric system (composition, miscibility, solubility), among other parameters. Despite the use of ionic liquids in the dissolution of biopolymers such as chitin [27-29], the use of chitin/IL in the production of porous matrices is still not widely studied in the literature. In our systems, processing of porous chitin-based materials was based on IL removal from the IL/chitin gels. Initially, the chitin gels were immersed in pure ethanol and a large amount of [bmim][Ac] was extracted, which was detected by a strong yellow colour in the liquid medium. Afterwards, the gels were submitted to Soxhlet extraction and successive steps of supercritical extraction. In the SCF process, the combination of $\mathrm{CO}_{2}$ and ethanol $(\mathrm{EtOH})$ in different ratios was used in order to optimize the [bmi$\mathrm{m}][\mathrm{Ac}]$ removal from the gels. The solubility of $\left[\mathrm{C}_{4} \mathrm{mim}\right][\mathrm{Ac}]$ has been reported to be very low $[30,31]$. Nonetheless, the presence of a co-solvent can enhance the IL solubility in the supercritical phase. As ethanol is both a good solvent for the ionic liquid and has a good solubility in carbon dioxide, it was considered to be the ideal co-solvent for the removal of [bmim] [Ac] from the constructs. In a subsequent step, the samples free of IL and dissolved in ethanol were dried under supercritical $\mathrm{CO}_{2}$. The chitin structures produced were completely opaque and shining white.

The excellent ionic conductivity of ILs up their decomposition temperature [3] enabled the evaluation of the efficiency of IL removal through conductivity measurements. Fig. $2 \mathrm{~A}$ demonstrates the progressive decrease in conductivity after the different steps of extraction process, indicating the efficiency of the IL removal. The findings also suggest that the association of the Soxhlet extraction with successive supercritical extractions with $\mathrm{CO}_{2}+$ ethanol resulted in increased efficiency of IL removal from the chitin-based materials. Moreover, no significant differences between the IL removal from the ChIL50 and ChIL70 samples were found.

It has also been found that, after IL removal by Soxhlet extraction, the drying procedure is a very important step in the construction of the porous chitin materials. The effect of the drying process on the chitin gels was evaluated. After Soxhlet extraction, different drying steps were analysed, i.e. freeze-drying, vacuum and supercritical drying. As can be seen in Fig. 2B, the obtained materials showed different morphologies that varied from a nonporous and rough surface to a well-defined porous structure. The drying with 
(A)
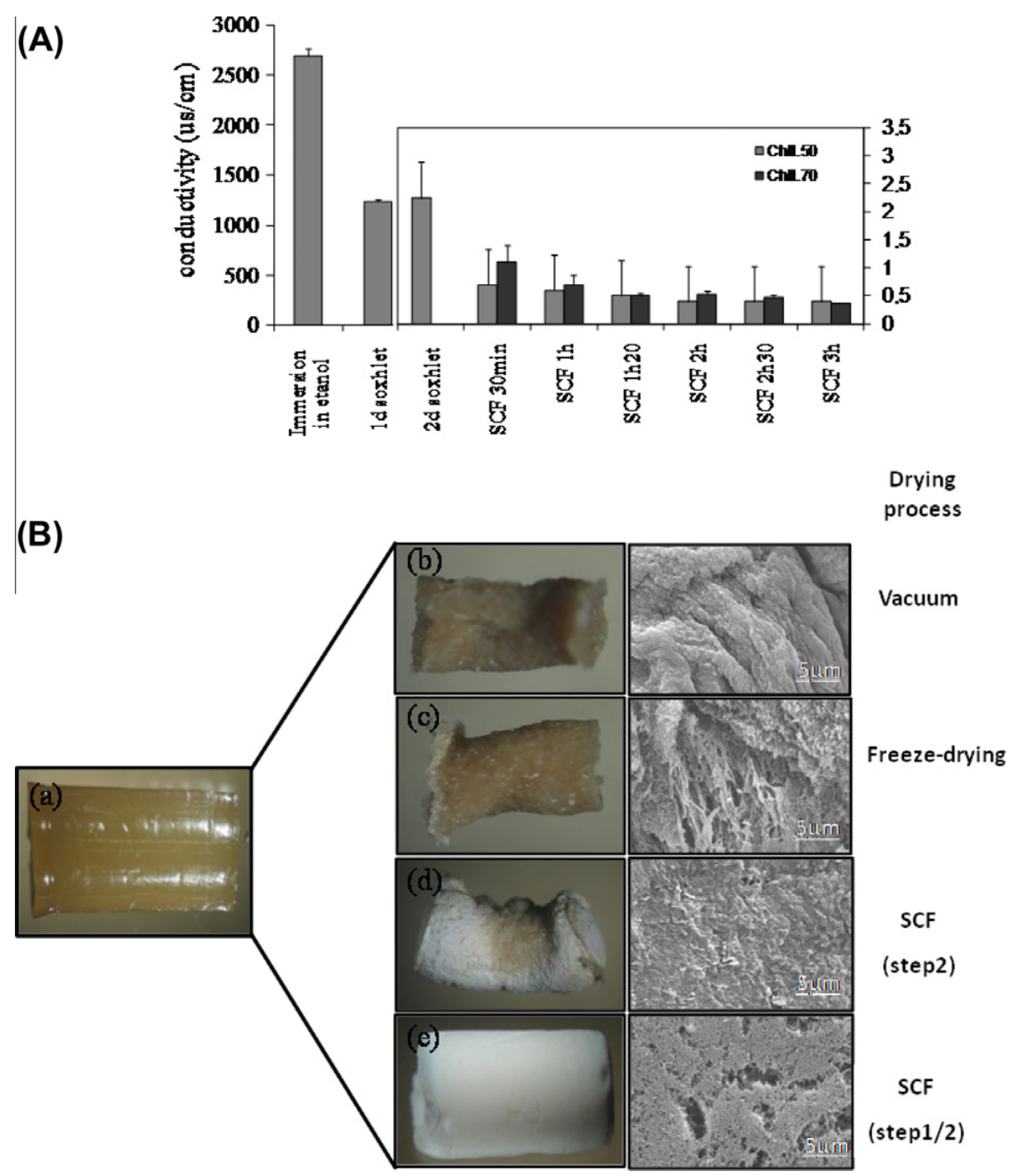

Fig. 2. (A) Conductivity measurements on aliquots of ethanol obtained after different steps of IL removal from ChIL gels. (B) Optical micrographs and SEM micrographs of the obtained chitin-based structures after different drying process: (a) as prepared, (b) Soxhlet extraction + vacuum, (c) Soxhlet extraction + freeze-drying, (d) Soxhlet extraction + SCF (step 2) and (e) Soxhlet extraction + SCF (step $1+$ step 2).

SCF (step1/2) seems to be superior to all the aforementioned methods in the production of porous chitin structures. The features of the produced structures can be associated to the presence of IL residues in the porous biomass, which affected the final morphology of material. Furthermore, due to the nature of chitin, the obtained structures are not as fragile as they appear. The open character of the structure was assured as the samples were processed continuously. Upon supercritical fluid extraction, the amount of IL remaining is very little and should therefore not affect the phase behaviour of the mixture greatly. However, at 100 bar and $70{ }^{\circ} \mathrm{C}$ the system is only in the supercritical state for the 50:50 $\left(\mathrm{CO}_{2}\right.$ : EtOH) mixture; the 70:30 mixture in this case is in a near-critical state, and no significant changes were observed due to this. There are few reports on $\mathrm{CO}_{2}$ :EtOH:IL mixtures, and the authors are aware that the introduction of one component to a mixture can sometimes change the phase equilibrium diagram of a particular system dramatically. The phase behaviour of the binary systems $\mathrm{CO}_{2}+[\mathrm{bmin}][\mathrm{Ac}]$ and $\mathrm{CO}_{2}+\mathrm{EtOH}$ were taken into consideration. Shifett and co-workers [30] report liquid-liquid separations with high carbon dioxide concentrations for binary solutions of $\mathrm{CO}_{2}+$ [bmin][Ac]; however, the low concentration of IL in the system during the supercritical extraction process and the high concentrations of ethanol may prevent this liquid-liquid separation, and we could in fact extract the ionic liquid from the matrix under the conditions of this study, as confirmed by the conductivity measurements carried out.

\subsection{Structural studies}

Fig. 3 shows the FTIR spectra of the [bmim][Ac], native chitin and porous chitin structures. In the spectrum of the native chitin (Fig. $3 \mathrm{~b}$ ), the peaks that appear in the amide region at 1660 , 1628 and $1557 \mathrm{~cm}^{-1}$ are assigned to the stretching of $\mathrm{C}=0$ groups bonded to $\mathrm{N}-\mathrm{H}$ groups of the adjacent chain, the stretching of the $\mathrm{C}=\mathrm{O}$ groups bifurcated by forming an additional hydrogen bond to the primary $\mathrm{OH}$ groups of the same chain, and $\mathrm{NH}$ deformations, respectively [32]. Comparing the spectra (Fig. 3a, $\mathrm{c}$ and d), we note that the absorption bands characteristic for [bmim][Ac] (Fig. 3a) could not be observed in the porous chitin structures' spectra, suggesting that [bmim][Ac] could be almost totally removed by Soxhlet extraction and SCF. Furthermore, the FTIR spectra of the native chitin and porous chitin structures (Fig. $3 \mathrm{~b}-\mathrm{d}$ ) are similar, indicating that the original chemical structure of the polymer was preserved. 


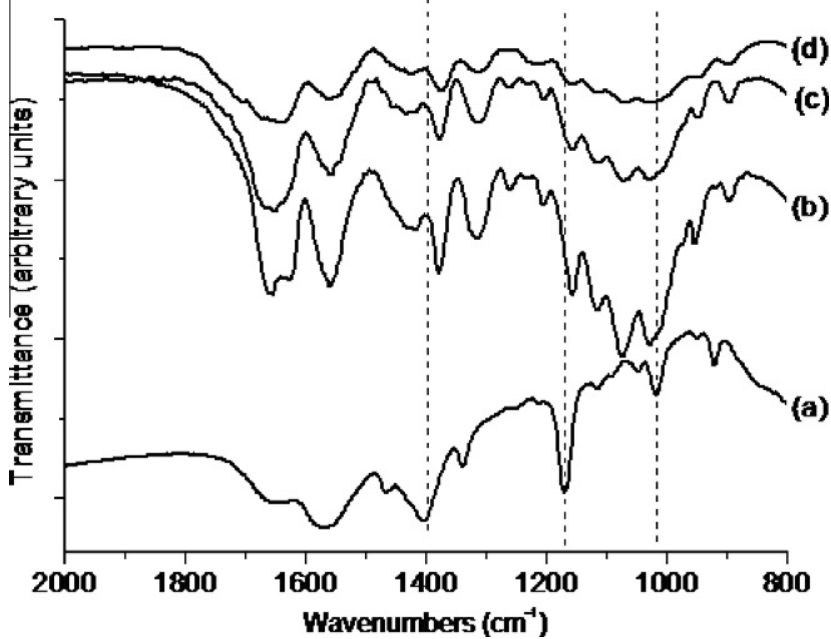

Fig. 3. FTIR spectra of [bmim][Ac] (a), chitin (b), ChIL50 (c) and ChIL70 (d).

\subsection{Morphology}

The morphology of both porous chitin structures (ChIL50 and ChIL70) was studied by SEM. Both samples displayed a heterogeneous porous formation (see Fig. 4), with pores around $1 \mu \mathrm{m}$ being observed. Comparing both samples (Fig. 4A and B), the ChIL50 has a nanofiber meshwork. The morphological features of the porous chitin structures may arise from the sensitivity of these samples to carbon dioxide during the extraction step. In fact, the combination of $\mathrm{CO}_{2}$ and ethanol in the 70/30 and 50/50 ratios used to optimize the [bmim][Ac] removal from the gels affected the material porosity (see Table 1), leading to a lower density of the chitin structures. Tsioptsias and co-workers [33] studied the influence of operating conditions on the porosity of the prepared nanostructured materials. In their work, the operating pressure during the supercritical drying showed a linear relation to the porosity of the matrices. However, in their work the ionic liquid was not extracted by means of supercritical carbon dioxide.

The nitrogen $\left(\mathrm{N}_{2}\right)$ adsorption results are illustrated in Fig. 5(A and $\mathrm{B}$ ) and summarized in Table 1 . The $\mathrm{N}_{2}$ adsorption-desorption isotherms of the chitin samples displayed in Fig. 5A are of type II of the IUPAC classification [15], indicating the essentially mesoporous nature of the materials (pore sizes between 2 and $50 \mathrm{~nm}$ ). These findings are further supported by the mesopore size distribution (Fig. 5B), which I wide in both samples.

The properties of the produced porous chitin matrices, e.g. high porosity, low density and the presence of small pores, can also be compared with the typical properties of aerogels. Tsioptsias et al. [34] described the supercritical point drying of gels as a common technique for aerogels, and also reported that the formation and properties of chitin aerogels depended on the preparation conditions and drying parameters [33,34]. However, in their studies, chitin was dissolved in dimethylacetamide/lithium chloride.

\subsection{XRD crystallinity}

The crystalline structure of chitin has been reported in previous works [32]. Its structure contains extended polymer chains, indicating an antiparallel arrangement of the chitin chain with strong intermolecular hydrogen bonding. XRD analysis was carried out upon the analysis of the effect regeneration of polymer. Fig. 6 shows the XRD patterns of chitin and regenerated materials after successive steps of the supercritical process. The XRD pattern of chitin has typical diffraction peaks at $2 \theta=9.3,19.3,23.2$ and
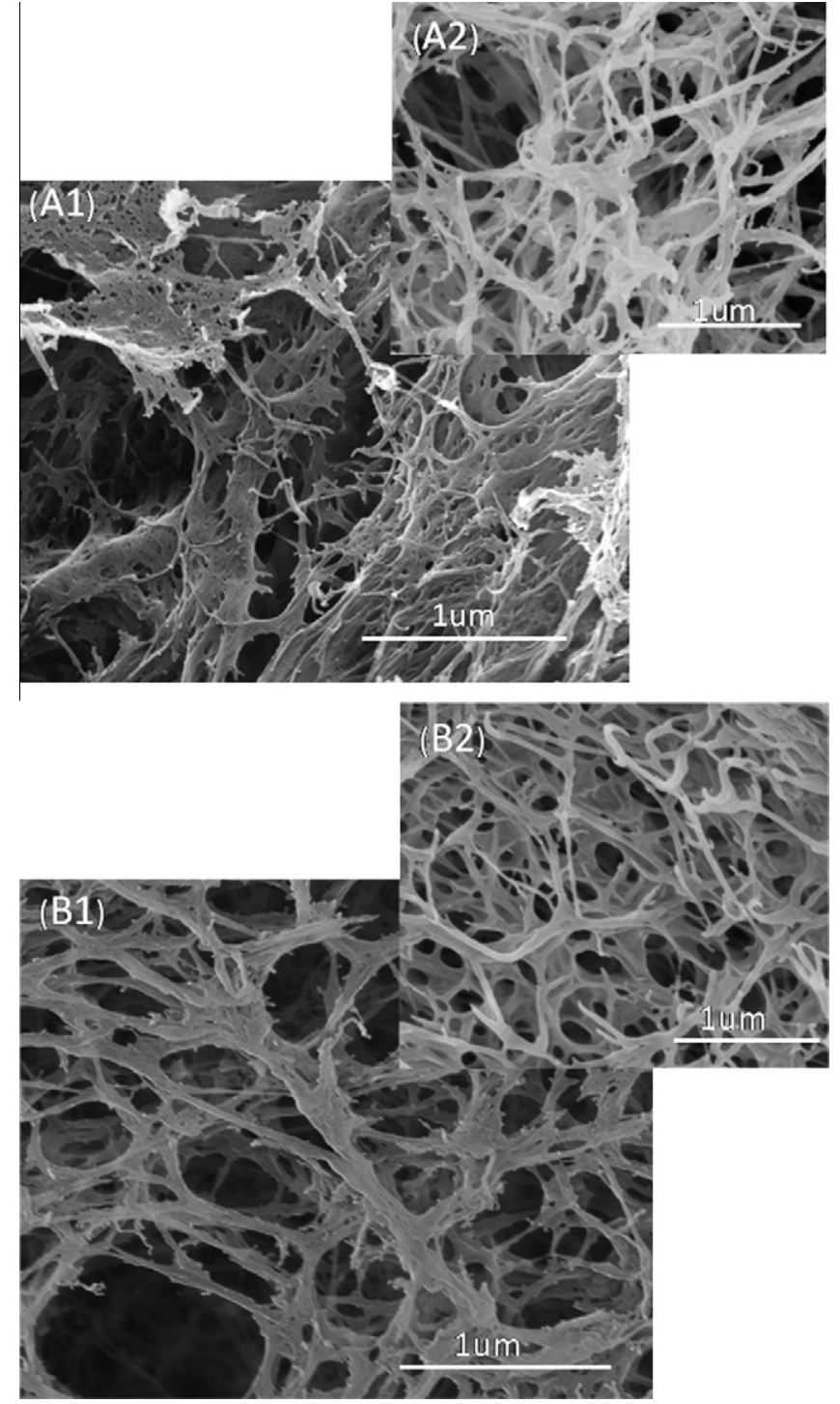

Fig. 4. SEM micrographs of the surface and cross-sections of porous chitin structures (ChIL), respectively: ChIL70 (A1, A2) and ChIL50 (B1, B2).

$26.3^{\circ}$, which are in accordance with previous findings [32,35]. The broad signal centred at $2 \theta=9.3^{\circ}$ can be attributed to glucosamine sequences, while the signal $2 \theta=19.3^{\circ}$ occurs due to $N$-acetylD-glucosamine monomers. Comparing the original chitin with the regenerated samples (Fig. 6A and B), it can be seen that the intensities of the referred peaks are reduced significantly, suggesting a decrease in crystallinity after IL removal. The crystallinity of the samples was calculated to be 31,17 and $18 \%$ for untreated chitin, ChIL70 and ChIL50, respectively. These values are in agreement with the previous observations of their XRD profiles. A possible explanation for these findings might be the possible breakdown of the inter- and intramolecular hydrogen bonds by [bmim][Ac] destroying the original crystalline zones of chitin, as reported in similar studies for cellulose [36].

\subsection{Mechanical properties}

The mechanical properties of the samples prepared were evaluated and compression tests were performed on ChIL70 and ChIL50 at a constant load. Fig. 7 shows a representative curve for the deformation behaviour of the porous chitin materials. The 
Table 1

Microstructural characteristics of porous chitin structures.

\begin{tabular}{lllll}
\hline Sample & $\mathrm{S}_{\mathrm{BET}}\left(\mathrm{m}^{2} \mathrm{~g}^{-1}\right)$ & $\begin{array}{l}\text { Bulk density } \\
\left(\mathrm{g} \mathrm{l}^{-1}\right)\end{array}$ & $\begin{array}{l}\text { Pore volume } \\
\left(\mathrm{cm}^{3} \mathrm{~g}^{-1}\right)\end{array}$ & $\begin{array}{l}\text { Mean } \\
\text { porosity }(\%)\end{array}$ \\
\hline ChIL70 & 108 & 0.039 & 0.22 & 90.2 \\
ChIL50 & 145 & 0.063 & 0.30 & 84.1 \\
\hline
\end{tabular}
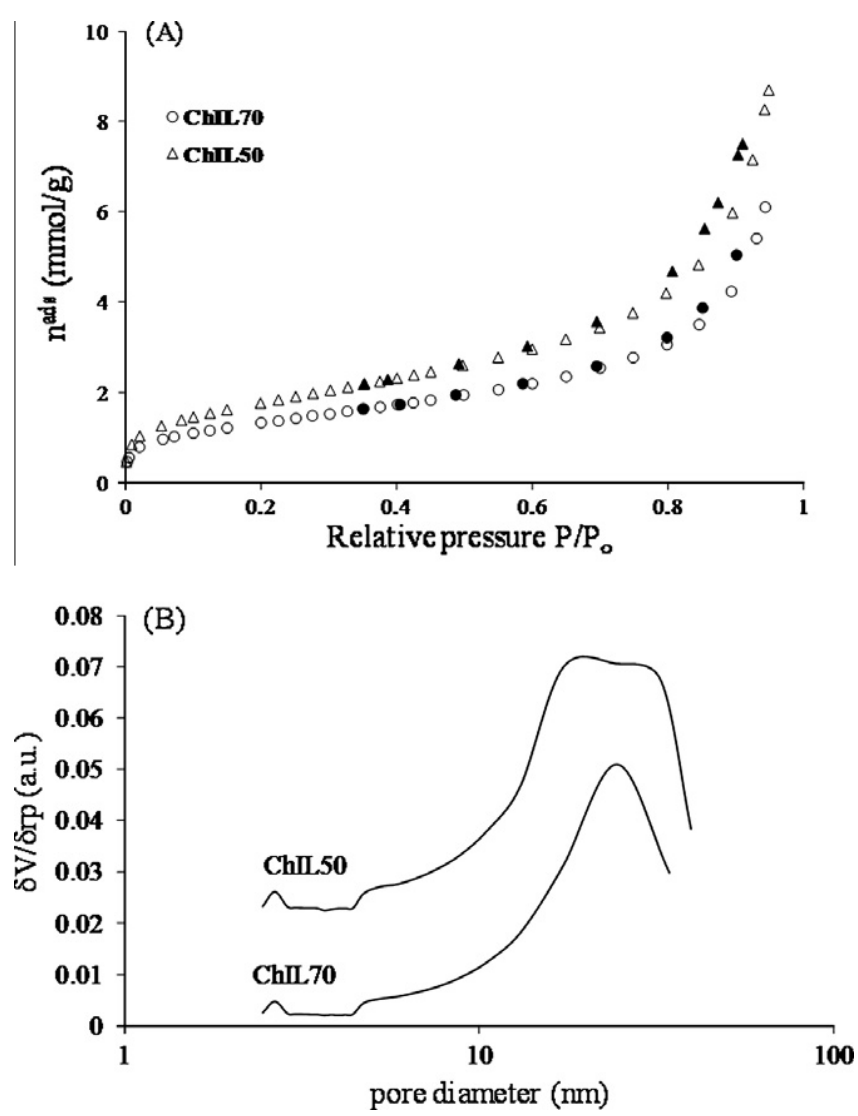

Fig. 5. (A) $\mathrm{N}_{2}$ adsorption-desorption isotherms of ChIL70 and ChIL50; (B) porous size distribution of ChIL50 and ChIL70. Closed symbols are desorption points.

mechanical response was evaluated in five replicates and the results are reproducible.

The mechanical response was similar for both materials. In the initial stage, the curves present a linear slope, which is indicative of the elastic behaviour of the samples for small strain values. The Young's modulus was calculated to be 3580 and $3620 \mathrm{kPa}$ for ChIL50 and ChIL70, respectively. Beyond elastic limit permanent deformation occurs. The point when the structure starts to collapse and the structure is irreversibly damaged corresponds to the yield strain of the material. For the chitin structures prepared, the yield strain is around 15\%. After this period, the stress increases slowly until the pore structure becomes compact and there is an increase in the mechanical stress of the material.

\subsection{Cytotoxicity assays}

Although ILs are usually considered as green solvents, toxicity studies have shown that many commonly used ILs exhibit a certain level of toxicity [37]. Taking this into account, and also the possible use of these materials in biomedical applications, a cytotoxicity assessment of both ChIL extracts and [bmim][Ac] solutions (prepared with different concentrations) was carried out as a preliminary approach to assessing their toxicity potential. The findings (data
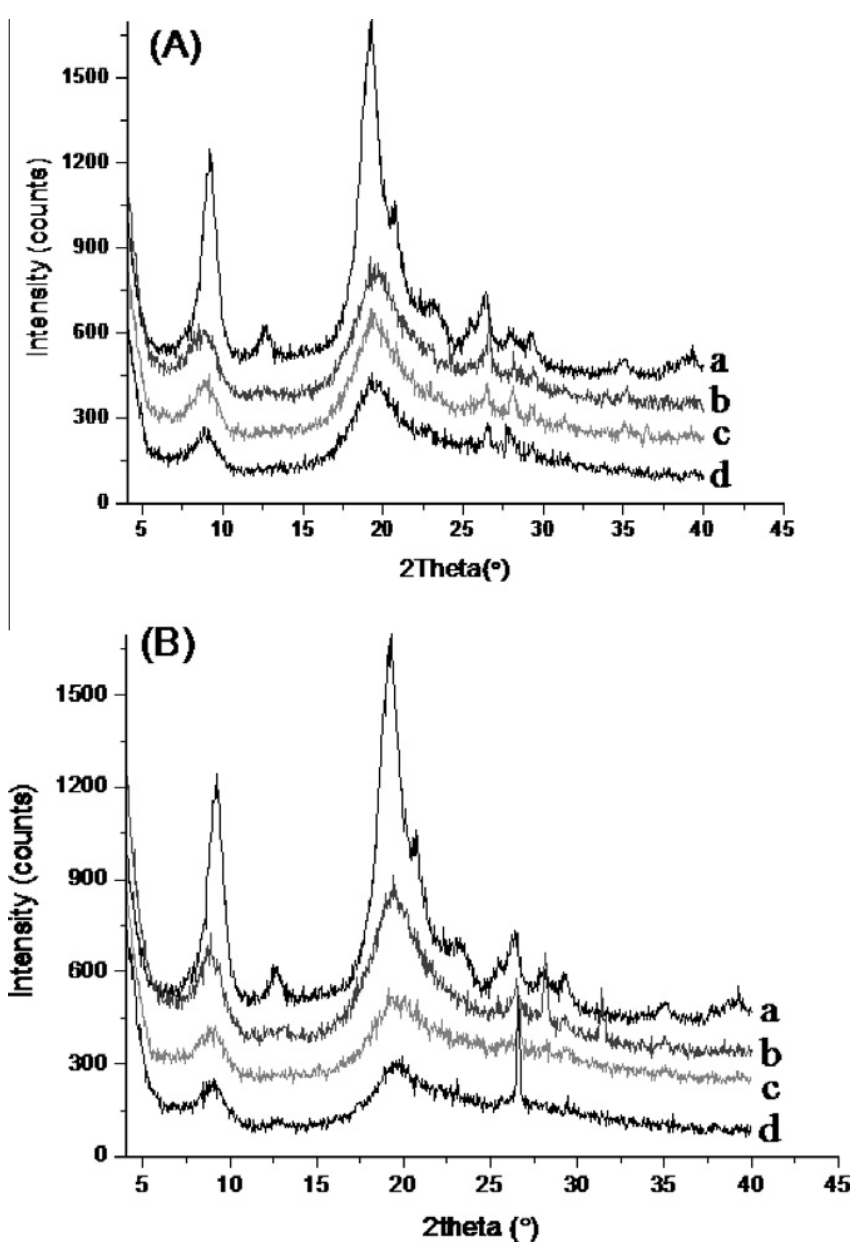

Fig. 6. XRD patterns of ChIL50 (A) and ChIL70 (B): native (a) and regenerated chitin after SCF $1 \mathrm{~h}$ (b), SCF $2 \mathrm{~h}$ (c) and SCF $3 \mathrm{~h}(\mathrm{~d})$.

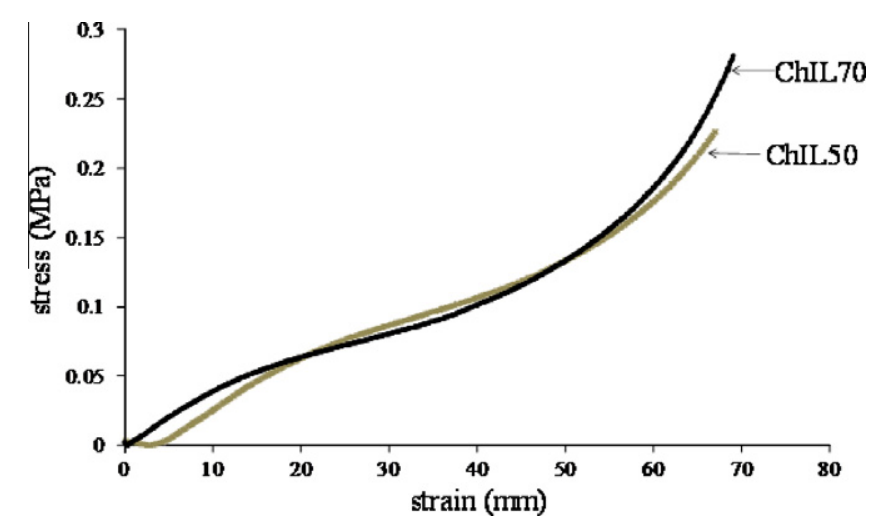

Fig. 7. Compressive stress-strain curves for ChIL70 and ChIL50 porous structures.

not shown) indicate that $\mathrm{L} 929$ cells have good cell viability in the IL solutions with low concentration (1.69 and $4.23 \times 10^{-3} \mathrm{~g} \mathrm{ml}^{-1}$ ). Moreover, the ChIL extracted presented good cell viability (about $120 \%$ ) when compared to tissue culture polystyrene. This demonstrates (i) the extremely low cytotoxicity levels of the ChIL porous structures and (ii) the efficiency of the [bmim][Ac] removal by the Soxhlet extraction and SCF process. Our findings indicate that the possible cytotoxic effect of ILs should be considered in the design of materials for biomedical applications. Nevertheless, the IL removal, total or partial performed during the processing of 
porous chitin structures can overcome this problem, promoting a decrease in their toxicity potential.

\section{Conclusion}

Ultralight porous chitin structures were successfully produced using an ionic liquid, 1-butyl-3-imidazolium acetate, as a solvent for chitin, and IL removal using Soxhlet extraction and successive steps of SCF process. The efficiency of the IL removal was confirmed through conductivity measurements. The developed chitin matrices showed interesting features, such as: (i) becoming less crystalline as a result of broken down hydrogen bonds in the chitin structure; (ii) possessing a wide mesoporous distribution; (iii) having very low density $\left(0.039-0.063 \mathrm{~g} \mathrm{l}^{-1}\right)$; and (iv) having a porosity of between $84 \%$ and $90 \%$. Most of these features were associated with the dissolution and regeneration of polymer in ethanol and to the IL removal process applied. Regarding the toxicity potential of both materials and [bmim][Ac] solutions, our findings indicated that ChIL extracts have good cell viability while cell viability in [bmim][Ac] solutions depends largely on concentration. It was also indicated that te [bmim] $[\mathrm{Ac}]$ removal, total or partial, performed during the processing of porous chitin structures promoted a reduction in the ionic liquid toxicity potential. These findings demonstrate that the formation of chitin gels in ILs and subsequent removal of [bmim] $[\mathrm{Ac}]$ from ChIL gels, using Soxhlet extraction and supercritical fluid technology, is a convenient way to prepare porous chitin structures. The matrices prepared are potential candidates for various relevant biomedical applications.

\section{Acknowledgements}

S.S.S. and A.R.C.D. thank the Portuguese Foundation for Science and Technology (FCT) for post-doctoral fellowships (SFRH/BPD/ $45307 / 2008$ and SFRH/BDP/34994/2007, respectively). This work was partially supported by the FCT (Project PTDC/QUI/68804/ 2006) and was carried out under the scope of the European NoE EXPERTISSUES (NMP3-CT-2004-500283).

\section{Appendix A. Figures with essential colour discrimination}

Certain figures in this article, particularly Figs. 2 and 7 are difficult to interpret in black and white. The full colour images can be found in the on-line version, at doi:10.1016/j.actbio.2010.09.041.

\section{References}

[1] El Seoud OA, Koschella A, Fidale LC, Dorn S, Heinze T. Applications of ionic liquids in carbohydrate chemistry: a window of opportunities. Biomacromolecules 2007;8:2629-47.

[2] Lu J, Yan F, Texter J. Advanced applications of ionic liquids in polymer science. Prog Polym Sci 2009;34:431-48.

[3] Anthony JL, Brennecke JF, Holbrey JD, Maginn EJ, Mantz RA, D RR, et al Physicochemical properties of ionic liquidsILs. In: Wasserscheid P, Welton T, editors. Ionic liquids in synthesis. Weinheim: Wiley-VCH Verlag; 2002.

[4] Reinhardt D, Ilgen F, Kralish D, Konig B, Kreisel G. Evaluation the greenness of alternative reaction media. Green Chem 2008;10:1170-81.

[5] Swatloski RP, Spear S, Holbrey JD, Rogers. Dissolution of cellulose with ionic liquids. J Am Chem Soc 2002;124:4974-5.

[6] Liebert T. Innovative concepts for the shaping and modification of cellulose. Macromol Symp 2008;262:28-38

[7] Quiang Z, Yan CM, Zhang B, Yang Y, Li RK. Novel all-cellulose ecocomposites prepared in ionic liquids. Cellulose 2009;16:217-26.
[8] Alves N, Mano J. Chitosan derivatives obtained by chemical modifications for biomedical and environmental applications. Int J Biol Macromol 2008;43: 401-14.

[9] Kurita K. Chitin and chitosan: functional biopolymers from marine crustaceans. Mar Biotechnol 2006;8:203-26.

[10] Pillai CKS, Paul W, Sharma CP. Chitin and chitosan polymers: chemistry, solubility and fiber formation. Prog Polym Sci 2009:34:641-78.

[11] Sashiwa H, Aiba SI. Chemically modified chitin and chitosan as biomaterials. Prog Polym Sci 2004;29:887-908.

[12] Anastas P, Warner J. Green chemistry: theory and practice. New York: Oxford University Press; 1998.

[13] Anderson CG, DePablo N, Romo CR, editors. Antarctic krill (Euphausia superba) as a source of chitin and chitosan. In: First International Conference on Chitin/ Chitosan, 1978. Cambridge, MA: MIT Sea Grant Program, 1978.

[14] Karageorgiou V, Kaplan D. Porosity of 3D biomaterial scaffolds and osteogenesis. Biomaterials 2005;26:5474-91.

[15] Rouquerol F, Rouquerol J, Sing KSW. Adsorption by powders and porous solids. London: Academic Press; 1999.

[16] Lukens W, Schmidt-Winkel P, Zhao D, Feng J, Stucky GD. Evaluating pore sizes in mesoporous materials: a simplified standard adsorption method and simplified Broekhoff-de Boer method. Langmuir 1999;15:5403-9.

[17] ISO/10993. Biological Evaluation of Medical Devices. Part 5. Test for Cytotoxicity, In Vitro Methods: 8.2 Test on Extracts. 1992.

[18] Gomes ME, Reis RL, Cunha AM, Blitterswijk CA, de Bruijn JD. Cytocompatibility and response of osteoblastic-like cells to starch-based polymers effect of several additives and processing conditions. Biomaterials 2001;22: 1911-7.

[19] Silva SS, Oliveira JM, Mano JF, Reis RL editors. Physicochemical charac terization of novel chitosan-soy protein/TEOS porous hydrids for tissue engineering applications. Advanced Materials Forum 2006.

[20] Duarte ARC, Mano JF, Reis RL. Supercritical fluids in biomedical and tissue engineering applications: a review. Int Mater Rev 2009;54:214-22.

[21] Duarte ARC, Mano JF, Reis RL. Preparation of chitosan scaffolds loaded with dexamethasone for tissue engineering applications using supercritical fluid technology. Eur Polym J 2009;45:141-8.

[22] Reverchon E, Cardea S, Rapuano C. A new supercritical fluid-based process to produce scaffolds for tissue replacement. J Supercrit Fluids 2008;45: 365-73.

[23] Tsivintzelis I, Pavlidou E, Panayiotou C. Porous scaffolds prepared by phase inversion using supercritical $\mathrm{CO}_{2}$ as antisolvent. I. Poly(l-lactic acid). J Supercrit Fluids 2007;40:317-22.

[24] Correlo V, Boesel L, Pinho E, Costa-Pinto A, Alves da Silva M, Battacharya M, et al. Melt-based compression moulded scaffolds from chitosan-polyester blends and composites: morphology and mechanical properties. J Biomed Mater Res A 2008;91A:489.

[25] Ma PX, Zhang R. Synthetic nano-scale fibrous extracellular matrix. J Biomed Mater Res 1999;46:60-72.

[26] Wei GB, Ma PX. Macroporous and nanofibrous polymer scaffolds and polymer/ bone-like apatite composite scaffolds generated by sugar spheres. J Biomed Mater Res A 2006;78A:306-15.

[27] Qin Y, Lu XM, Sun N, Rogers RD. Dissolution or extraction of crustacean shells using ionic liquids to obtain high molecular weight purified chitin and direct production of chitin films and fibers. Green chem 2010;12:968-71.

[28] Wu Y, Sasaki T, Irie S, Sakurai K. A novel biomass-ionic liquid platform for the utilization of native chitin. Polymer 2008;49:2321-7.

[29] Xie H, Zhang S, Li S. Chitin and chitosan dissolved in ionic liquids as reversible sorbents of $\mathrm{CO}_{2}$. Green chem 2006;8:630-3.

[30] Shifett M, Kasprzak D, Junk C, Yokozeki A. Phase behaviour of carbon dioxide + (bmin) $(A c)$ mixtures. J Chem Thermodyn 2008;40:25-31.

[31] Yaokozeki A, Shifett M, Junk C, Grieco L, Foo T. Physical and chemical absorptions of carbon dioxide in room temperature ionic liquids. J Phys Chem B 2008;112:16654-63.

[32] Cardenas G, Cabrera G, Taboada E, Miranda S. Chitin characterization by SEM, FTIR, XRD, and $13 \mathrm{C}$ cross polarization/mass angle spinning NMR. J Appl Polym Sci 2004;93:1876-85.

[33] Tsioptsias C, Michailof C, Stauropoulos G, Panayiotou C. Chitin and carbon aerogels from chitin alcogels. Carbohydr Polym 2009;76:535-40.

[34] Tsioptsias C, Panayiotou C. Foaming of chitin hydrogels processed by supercritical carbon dioxide. J Supercrit Fluids 2008;47:302-8.

[35] Sagheera F, Al-Sughayerb M, Muslima S, Elsabeec M. Extraction and characterization of chitin and chitosan from marine sources in Arabian Gulf. Carbohydr Polym 2009;77:410-9.

[36] Singh S, Simmons B, Vogel K. Visualization of biomass solubilization and cellulose regeneration during ionic liquid pretreatment of switchgrass. Biotechnol Bioeng 2009;104:68-75.

37] Zhao D, Liao Y, Zhang Z. Toxicity of ionic liquids. Clean 2007;35:42-8. 